\title{
$\begin{array}{ll}\text { Research Square } & \text { Preprints are preliminary reports that have not undergone peer review. } \\ \text { They should not be considered conclusive, used to inform clinical practice, } \\ \text { or referenced by the media as validated information. }\end{array}$
}

\section{Disseminated Coccidioidomycosis of the Knee Joint Requiring Synovectomy and Arthrotomy}

\section{Kavina Patel ( $\sim$ patelk11@livemail.uthscsa.edu )}

University of Texas Health Science Center at San Antonio https://orcid.org/0000-0003-0248-374X

\section{Farhan Ahmad}

University of Texas Health Science Center at San Antonio

\section{J.C. De Leon}

University of Texas Health Science Center at San Antonio

Frank Buttacavoli

University of Texas Health Science Center at San Antonio

\section{Case report}

Keywords: Coccidioidomycosis, valley fever, knee, fungal infection, orthopaedic surgery

Posted Date: February 24th, 2020

DOI: https://doi.org/10.21203/rs.2.24288/v1

License: (9) This work is licensed under a Creative Commons Attribution 4.0 International License. Read Full License 


\section{Abstract}

\section{Background}

This article presents a case of an immunocompetent patient from an endemic area diagnosed with disseminated coccidioidomycosis of the knee joint, which was unusually severe and required operative management.

\section{Case presentation}

A 49-year-old patient with a history of pulmonary coccidiomyocosis presented with right knee pain and multiple symptomatic abscesses beneath the suprapatellar and infrapatellar fat pads. Arthocentesis and culture confirmed the infection, and open synovectomy, arthrotomy and drainage of the infection was performed without complication.

\section{Conclusions}

Disseminated coccidioidomycosis is an uncommon fungal infection that may involve joints and become refractory to pharmacotherapy. Management may require surgical intervention along with infectious disease consultation and close follow-up. Patients from endemic regions should be evaluated with a comprehensive history for this disease.

\section{Background}

Coccidioidomycosis is a fungal infection caused by the Coccidoides immitis or Coccidioides posadasii species that are endemic to Southwestern United States, Mexico, and South America.[1] The fungi are known to survive in arid and alkaline environments.[2] Most infections are established through inhalation of aerosolized arthrospores, but may less commonly inoculate patients through direct cutaneous contact.

Initial pulmonary infections are typically asymptomatic or present with mild lower respiratory symptoms and resolve spontaneously.[3, 4] Symptomatic cases will present similar to community-acquired pneumonia, with flu-like symptoms and a possible rash-also known as "Valley Fever". There are no pathognomonic chest imaging findings.

Hematogenous extra-pulmonary systemic dissemination may present in $1 \%$ of infections and may involve the integumentary and musculoskeletal systems, with comparatively more cases occurring in older individuals and in males.[5, 6] About $10-30 \%$ of disseminated coccidioidomycosis cases will have musculoskeletal involvement.[3] Epidemiologic studies posit that about 1500 Americans per year are infected with disseminated coccidioidomycosis. $[7,8]$ Due to the low prevalence of this form of the disease, clinicians surgeons should maintain a high index of suspicion and obtain comprehensive histories for patients from endemic areas, especially those with a history of the infection.[9] 
In this article, we present a case of an immunocompetent patient from an endemic area diagnosed with disseminated coccidioidomycosis of the knee joint, which required operative management. The patient's risk of exposure was determined by his endemic residence and occupation history. The rarity of this presentation of coccidioidomycosis is noteworthy and an opportunity for clinicians to review the diagnosis and treatment of disseminated coccidioidomycosis.

\section{Case Presentation}

A 49-year-old male oil field worker from West Texas with a past history of gastroesophageal reflux disease, hypertension, obstructive sleep apnea, and obesity presented with a painful right knee effusion for at least one year. He had a history of coccidioidomycosis pneumonia 15 months prior, which presented as left lower lobe nodular infiltrates on radiographs, along with fever, chills, dyspnea, and malaise. The patient's pulmonary coccidioidomycosis was treated with fluconazole for 12 months without much response, and he concurrently developed worsening right knee pain. Infectious disease consultants subsequently placed the patient on itraconazole for 3 months for disseminated coccidioidomycosis infection, which abated his pulmonary symptoms but not his knee pain. At time of presentation to orthopaedics, the lungs were clear, with no infiltrates or pleural effusions present on imaging.

Physical exam demonstrated right knee effusion and palpable soft tissue mass in the lateral suprapatellar and lateral infra-patellar regions (Figure 1A and 1B). The patient tolerated an axial load and was able to ambulate with an antalgic gait. His knee range of motion with discomfort was from full extension to 110 degrees of flexion. He had no pain with examination of his hip or his other joints at initial presentation. Knee joint aspirate demonstrated a yellow hazy fluid, elevated white blood cell count $\left(12,579 / \mathrm{mm}^{3}\right)$ with $56 \%$ polynuclear cells and $44 \%$ monocytes. Fungal culture was positive for Coccidioides immitis and Coccidioides posadasii. Serum serologies demonstrated positive Coccidioides IgG (9.0) and IgM (2.8) antibodies. Serum complement fixation was elevated at 1:1024. Cerebrospinal fluid demonstrated positive Coccidioides $\lg G(2.9)$, and negative $\lg M(0)$.

Radiographs of the right knee showed a large joint effusion without underlying osseous abnormality (Figure 2A, 2B, 2C). Magnetic Resonance Imaging (MRI) of the knee confirmed extensive synovial thickening and enhancement throughout the right knee joint compatible with synovitis (Figure 3A, 3B, 3C). Additionally, subcutaneous abscesses superficial to the quadriceps and patellar tendons were identified.

The patient provided informed consent to undergo a right knee open synovectomy, biopsy, arthrotomy and drainage. During surgery, superficial abscesses (measuring $4 \times 4 \mathrm{~cm}$ and $2 \times 2 \mathrm{~cm}$ ) with extensive synovial thickening were found with no knee joint involvement. The patient tolerated surgery well and encountered no intraoperative complications. The wound was primarily closed with an incisional vacuum placed. 
Histopathologic examination of drained fluid from surgery revealed spherules with endospores suggestive of Coccidioides (Figure 4A and 4B).

Postoperatively, an infectious disease consultant placed the patient on liposomal amphotericin B (AmB), vancomycin, metronidazole, and cefepime. The incisional vacuum was removed on postoperative day 2 . At discharge, the wound was healing well and there was no evidence of surgical site infections. The patient was given a hinged knee brace but was advised to spend time without it to work on increasing his range of motion. The patient was also put on $200 \mathrm{mg}$ itraconazole twice a day at discharge.

The patient continued following up with orthopaedics at 3 months and 6 months. At the 6-month followup visit, the patient endorsed improved functional status and was ambulating without assistance. Physical examination showed a well-healed surgical scar with no signs of reinfection or drainage. Knee range of motion had returned to greater than 90 degrees flexion and full extension (Figure 5A and 5B). Infectious disease specialists decided to continue the itraconazole regimen indefinitely, periodically following the patient for his chronic coccidioidomycosis infection.

\section{Discussion And Conclusion}

This case presents disseminated coccidioidomycosis infection of the knee joint with periarticular synovial involvement and abscess formation. Skeletal coccidioidomycosis typically presents as a chronic synovitis, arthritis, or osteomyelitis of axial structures, but the knee is an appendicular joint also known to become infected. Periarticular manifestations include tenosynovitis, tendinitis, and effusion, along with concomitant soft tissue infections. Joint space destruction can also happen in the later stages of the disease if left untreated.[10]

Accurate diagnosis of this disease, especially in the immunocompetent, requires a high index of suspicion, especially in those from endemic areas or with a history of the disease. Thorough history and physical examination should elucidate travel to, or residence in endemic areas, as well as any past respiratory illness that can predate skeletal manifestations of coccidioidomycosis. Epidemiologic studies indicate that the disseminated form of this disease follows within a year of acute respiratory infection. Pregnant women and patients of Hispanic, African, or Filipino descent are at increased risk. Other risk factors include advanced age, diabetes, and pre-existing cardiopulmonary conditions. It should be noted that although immunocompromised patients are at the highest risk of serious disseminated infections, immunocompetent patients are still susceptible.[11]

Fungal etiologies should remain on the clinician's differential for patients presenting with signs of joint inflammation, such as swelling, redness, and pain-especially in endemic regions. Arthrocentesis should be performed on joints to assist with diagnosis, and a survey of other joints should be performed to assess for simultaneous, multifocal areas of infection. Additionally, any laboratory receiving specimens with potential coccidiomycosis should be alerted, because the organism is a biosafety level 3 pathogen. The gold standard for diagnosis of skeletal coccidiomycosis is culture and/or serological testing. 
Radiographs and/or MRI can be useful for assessing the extent of involvement and formulating surgical plans. However, imaging can introduce pitfalls as well. On MRI, coccidiomycosis of the bone can present as hyperintense lesions that may be misconstrued as metastatic bone disease. The differential diagnosis for these lesions based on imaging include other fungal and granulomatous diseases or osteosarcomas and other malignancies.[12]

Operative management of disseminated coccidioidomycosis typically requires incision and debridement and resection of disease sequestra. Necrotic tissue should be removed with healthy margins and the reduction of pathogenic burden is the mainstay of treatment.[13] In contrast to disseminated tuberculosis, debridement of coccidiomycosis is indicated.

Following operative removal of the fungal pathogen burden, the initiation of culture-specific antifungal pharmacotherapy, such as AmB, ketoconazole, fluconazole, or itraconazole is needed.[14] Consult from an infectious disease physician is recommended for assistance with the management of antibiotic choice and duration of treatment. Our patient was initially treated with fluconazole followed by itraconazole due the presence of significant pulmonary nodular infiltrates in the left lower lobe. Once the patient presented with disseminated infection, itraconazole was continued and AmB was administered postoperatively per Infectious Disease Society of America and the Centers for Disease Control and Prevention guidelines.[14]

Lifelong suppressive treatment is often required for patients, and it should be noted that lipid formulations of drugs may be more costly, less accessible, and lead to insurance denials if an alternative drug is available.[15] In general, itraconazole is preferred for patients with bone and joint manifestations of disseminated coccidioidomycosis infection. Antibody titers can be employed as needed to follow treatment progress. Research on future therapies appears promising and includes the development of a live attenuated immunization against $C$. immitis, which has been successful in murine in vitro experiments.[16, 17]

Clinicians should consider disseminated coccidioidomycosis infection in patients presenting with a painful knee effusion, past respiratory illness, and/or travel to endemic regions.

\section{List Of Abbreviations}

Magnetic Resonance Imaging (MRI)

Amphotericin B (AmB)

\section{Declarations}

Ethics approval and consent to participate: Not applicable 
Consent for publication: Per the Declaration of Helsinki, the patient was informed and consented to allow photographs and case data to be submitted for publication.

Availability of data and materials: The datasets used and/or analysed during the current study are available from the corresponding author on reasonable request.

Competing interests: The authors declare that they have no competing interests.

Funding: No funding sources

Authors' contributions: KP and FA analyzed and interpreted the patient's case, and were major contributors in writing the manuscript. JDL and FB provided care to the patient, and provided modifications and thorough feedback to the manuscript.

Acknowledgements: Not Applicable

\section{References}

1. Hirschmann JV. The early history of coccidioidomycosis: 1892-1945. Clin Infect Dis 2007; 44:1202-7.

2. Hector RF, Laniado-Laborin R. Coccidioidomycosis-a fungal disease of the Americas. PLoS Med 2005; 2:e2.

3. Ho L, Schnall S, Schiller F, Holtom P. Metacarpal coccidioidal osteomyelitis. Am J Orthop (Belle Mead NJ) 2011; 40:34-6.

4. Smith CE, Beard RR, et al. Varieties of coccidioidal infection in relation to the epidemiology and control of the diseases. Am J Public Health Nations Health 1946; 36:1394-402.

5. Galgiani JN. Coccidioidomycosis: a regional disease of national importance. Rethinking approaches for control. Ann Intern Med 1999; 130:293-300.

6. Tan LA, Kasliwal MK, Nag S, O'Toole JE, Traynelis VC. Rapidly progressive quadriparesis heralding disseminated coccidioidomycosis in an immunocompetent patient. J Clin Neurosci 2014; 21:104951.

7. Fiese MJ. Recent experiences in the treatment of disseminated coccidioidomycosis. Stanford Med Bull 1955; 13:91-7.

8. Sandoval JJ, Shank JR, Morgan SJ, Agudelo JF, Price CS. Midfoot coccidioidal osteomyelitis. A case report and review of the literature. J Bone Joint Surg Am 2006; 88:861-5.

9. Desai SA, Minai OA, Gordon SM, O'Neil B, Wiedemann HP, Arroliga AC. Coccidioidomycosis in nonendemic areas: a case series. Respir Med 2001; 95:305-9.

10. Weisenberg SA. Coccidioides immitis septic knee arthritis. BMJ Case Rep 2018; 2018.

11. Campbell M, Kusne S, Renfree KJ, et al. Coccidioidal Tenosynovitis of the Hand and Wrist: Report of 9 Cases and Review of the Literature. Clin Infect Dis 2015; 61:1514-20. 
12. Zeppa MA, Laorr A, Greenspan A, McGahan JP, Steinbach LS. Skeletal coccidioidomycosis: imaging findings in 19 patients. Skeletal Radiol 1996; 25:337-43.

13. Bried JM, Galgiani JN. Coccidioides immitis infections in bones and joints. Clin Orthop Relat Res 1986:235-43.

14. Stockamp NW, Thompson GR, 3rd. Coccidioidomycosis. Infect Dis Clin North Am 2016; 30:229-46.

15. Galgiani JN, Catanzaro A, Cloud GA, et al. Comparison of oral fluconazole and itraconazole for progressive, nonmeningeal coccidioidomycosis. A randomized, double-blind trial. Mycoses Study Group. Ann Intern Med 2000; 133:676-86.

16. Cassone A, Casadevall A. Recent progress in vaccines against fungal diseases. Curr Opin Microbiol 2012; 15:427-33.

17. Crum NF, Lederman ER, Stafford CM, Parrish JS, Wallace MR. Coccidioidomycosis: a descriptive survey of a reemerging disease. Clinical characteristics and current controversies. Medicine (Baltimore) 2004; 83:149-75.

\section{Figures}

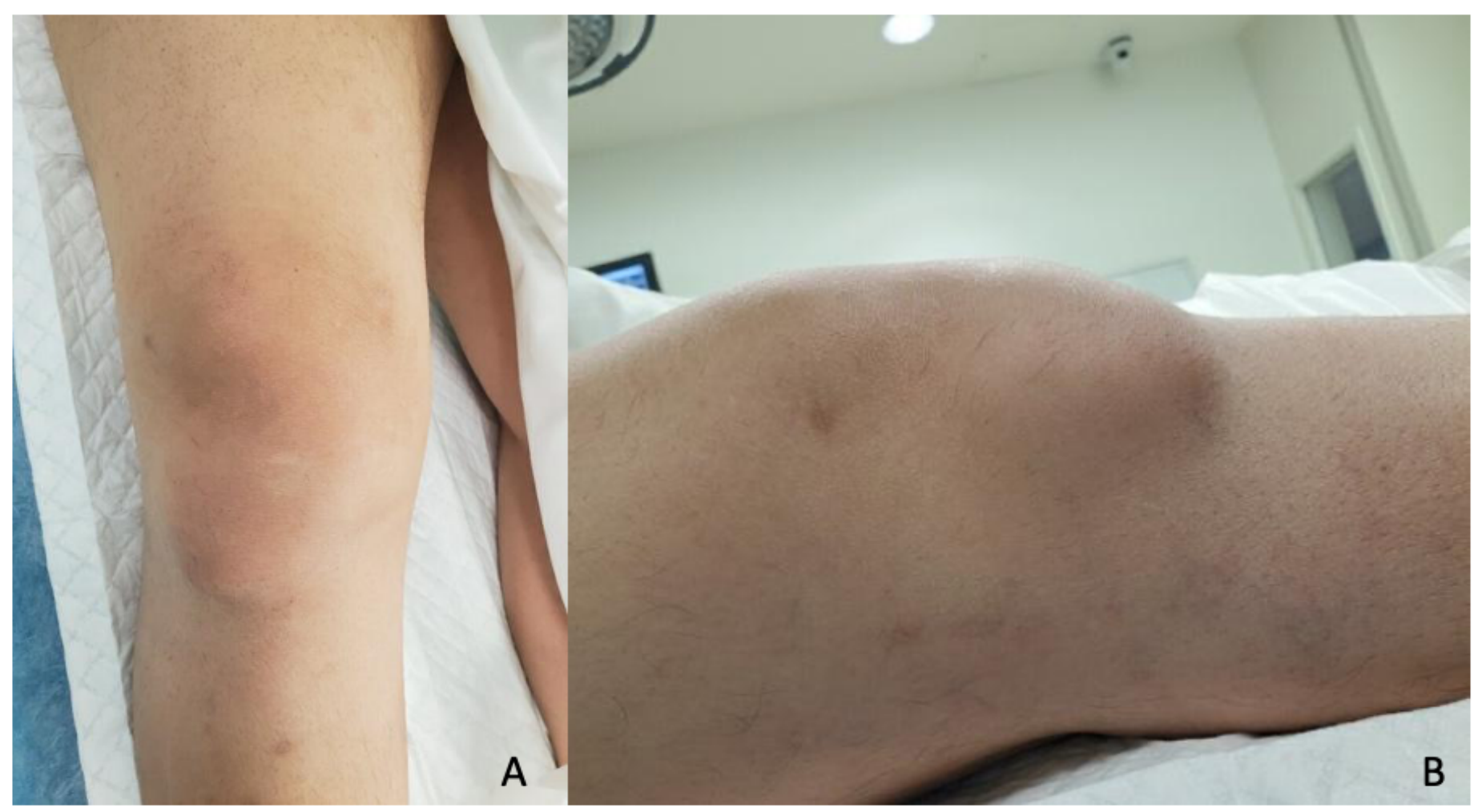

\section{Figure 1}

Figure 1A and 1B. Right knee effusion and palpable soft tissue mass in the lateral supra-patellar and lateral infra-patellar regions. 


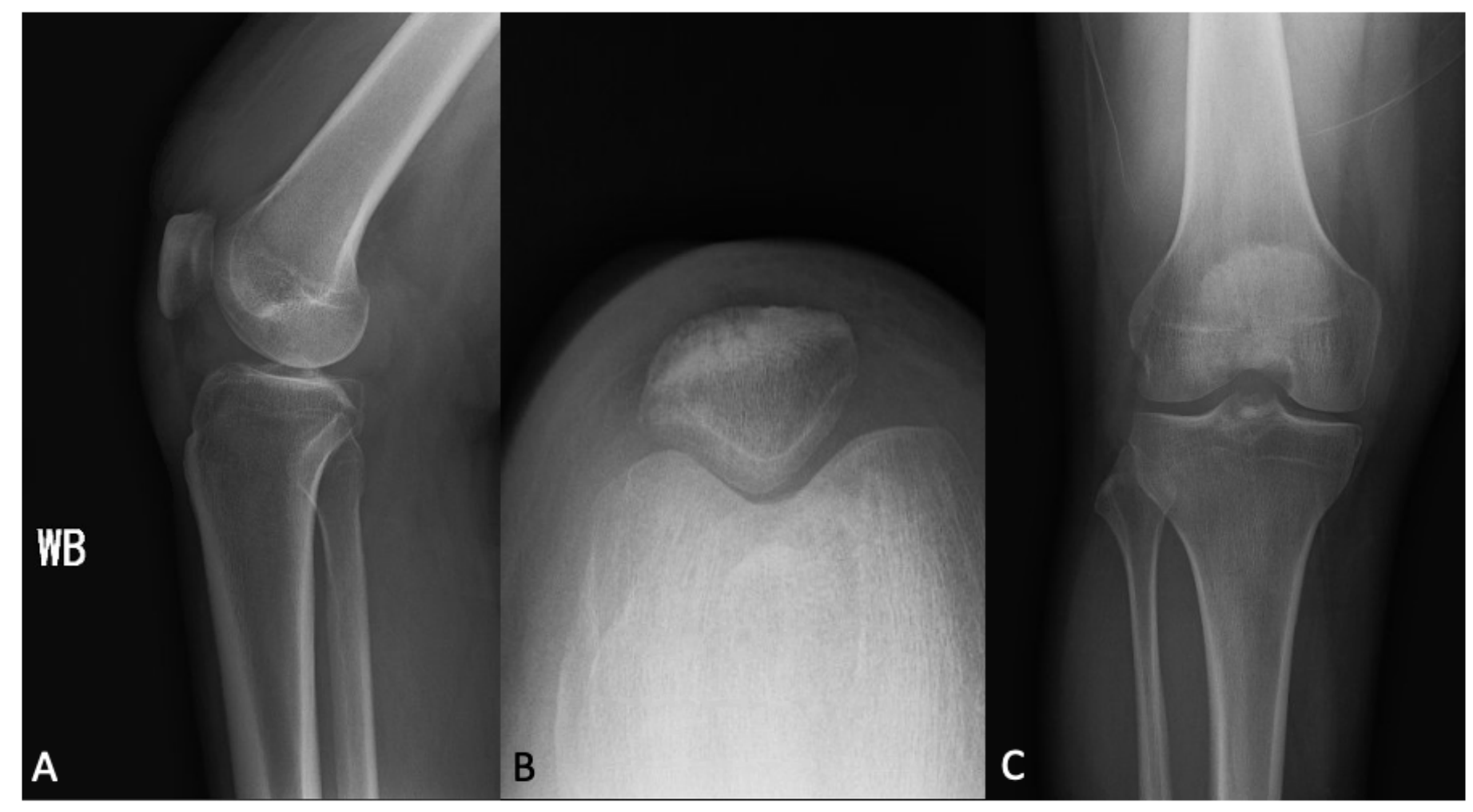

Figure 2

Figure 2A, 2B, 2C. Large right knee effusion with underlying osseous abnormality.

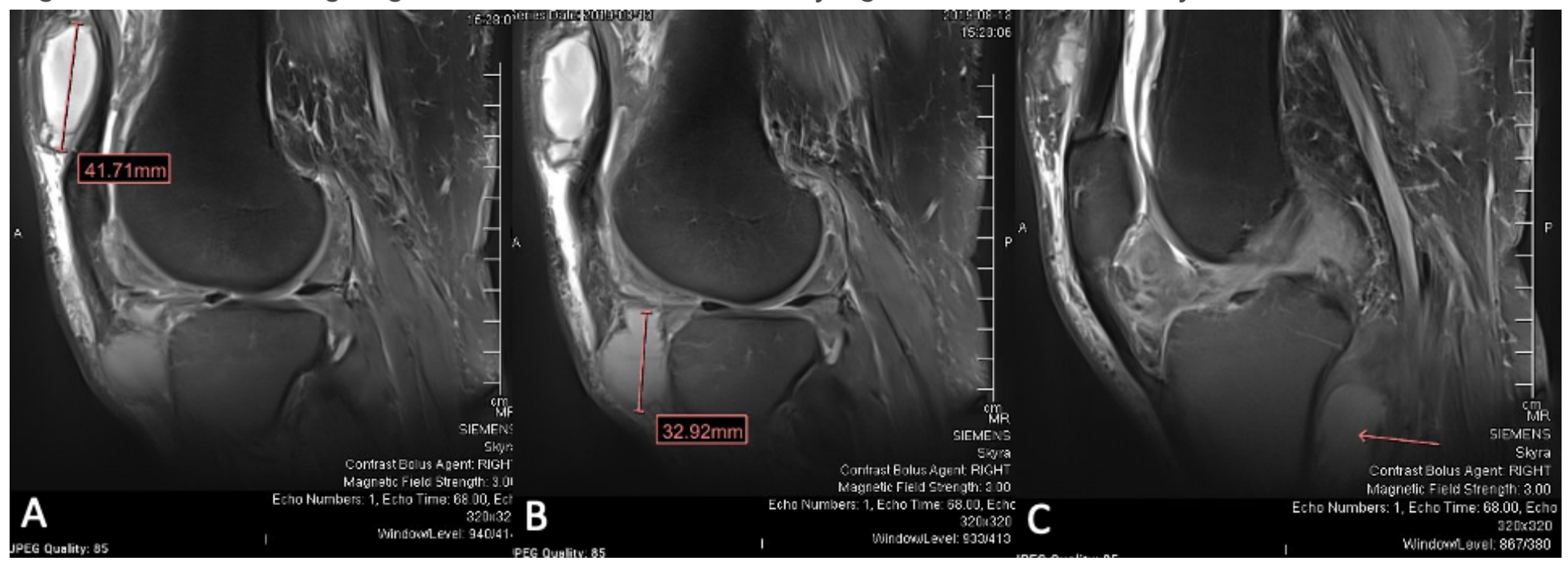

Figure 3

Figure 3A, 3B, 3C. MRI demonstrating a very large right knee joint effusion containing debris. There is an extensive synovial thickening and enhancement through the right knee joint compatible with synovitis. 展

Figure 4 
Figure 4A and 4B. Three dimensional view of spherules in Gram stain. 4A: A spherule with endospores 4B: A spherule with double walls.

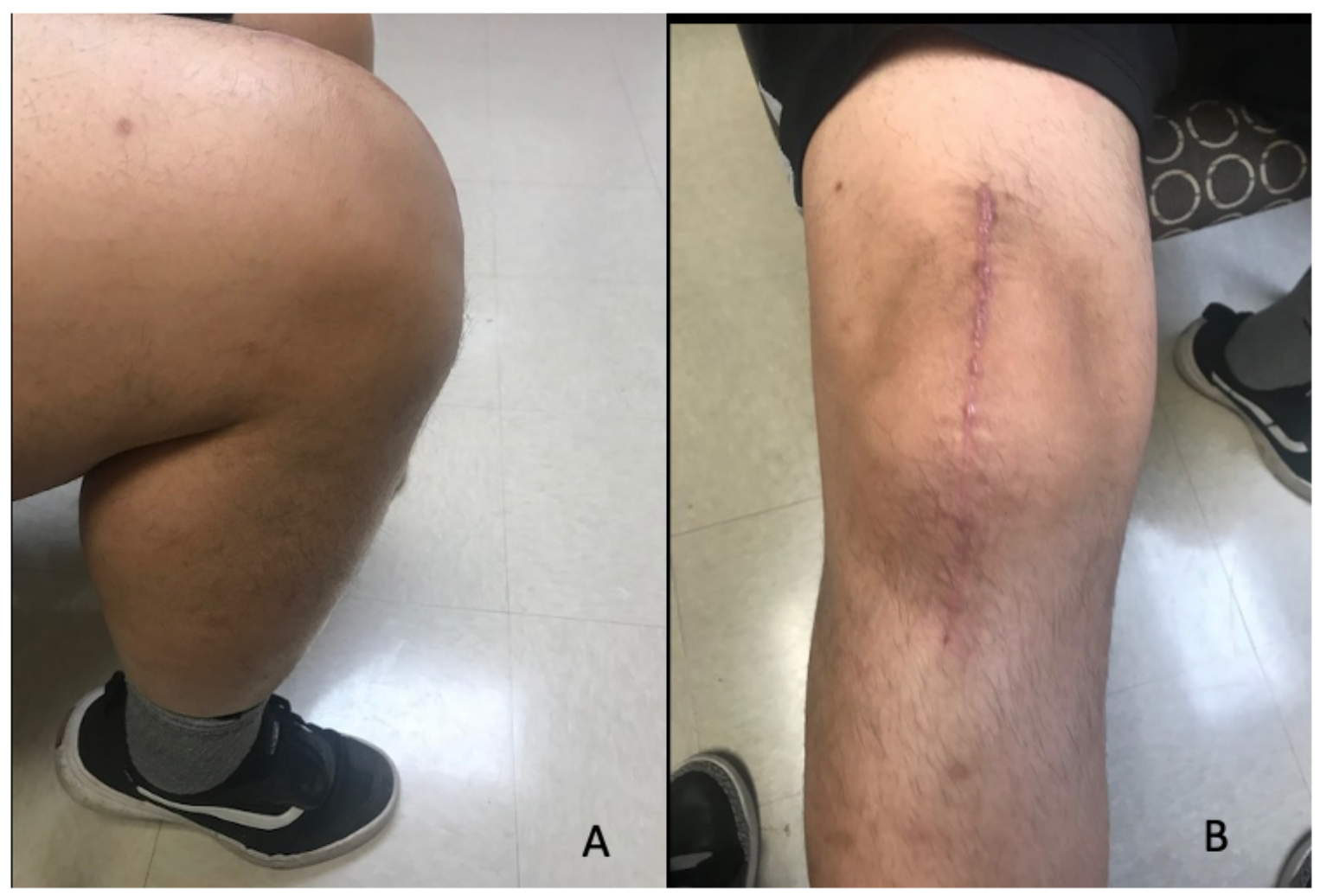

\section{Figure 5}

Figure 5A and 5B. Postoperative follow-up examination at 6 months. 5A: Knee flexion of approximately 110 degrees. 5B: Well-healing surgical scar with no evidence of reinfection. 